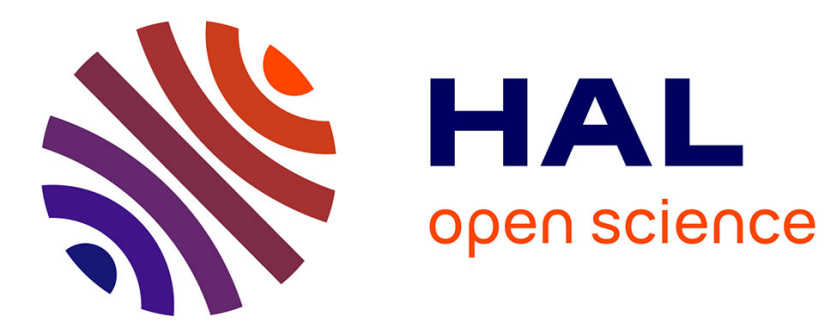

\title{
Non-classical effects in collective double optical resonance
}

\author{
N.N. Bogolubov, A.S. Shumovsky, Tran Quang
}

\section{To cite this version:}

N.N. Bogolubov, A.S. Shumovsky, Tran Quang. Non-classical effects in collective double optical resonance. Journal de Physique, 1987, 48 (11), pp.1925-1931. 10.1051/jphys:0198700480110192500 . jpa-00210634

\section{HAL Id: jpa-00210634 https://hal.science/jpa-00210634}

Submitted on 1 Jan 1987

HAL is a multi-disciplinary open access archive for the deposit and dissemination of scientific research documents, whether they are published or not. The documents may come from teaching and research institutions in France or abroad, or from public or private research centers.
L'archive ouverte pluridisciplinaire HAL, est destinée au dépôt et à la diffusion de documents scientifiques de niveau recherche, publiés ou non, émanant des établissements d'enseignement et de recherche français ou étrangers, des laboratoires publics ou privés. 


\title{
Non-classical effects in collective double optical resonance
}

\author{
N. N. Bogolubov (Jr.), A. S. Shumovsky and Tran Quang (*) \\ Laboratory of theoretical physics, Joint Institute for Nuclear Research, P.O. Box 79, 101000, Moscow, \\ U.S.S.R.
}

(Reçu le 24 février 1987, révisé le 9 juin 1987, accepté le 16 juin 1987)

\begin{abstract}
Résumé. - On discute de la compression des fluctuations et de la violation de l'inégalité de Cauchy-Schwarz pour le champ de fluorescence dans le processus de double résonance collective.
\end{abstract}

Abstract. - The squeezing and violation of the Cauchy-Schwarz inequality in the fluorescent field of collective double resonant process are discussed.

\section{Introduction.}

In many nonlinear systems involving the interaction between light and medium, some non-classical effects are observed. The reviews of such nonclassical effects in optics are given in papers by Loudon [1], Paul [2] and Walls [3]. The most well-known example of these effects is the photon antibunching which was observed in the experimental works by Kimble et al. [4-5].

Another example of non-classical effects is the violation of the Cauchy-Schwarz (C-S) inequality which was observed in the work by Clauser [6]. The violation of the C-S inequality was also predicted in the two-photon laser [7] and parametric amplifier [8].

In recent years a large number of theoretical and experimental works are concentrated on the problem of squeezed states of light [9-18, 31-32], which were

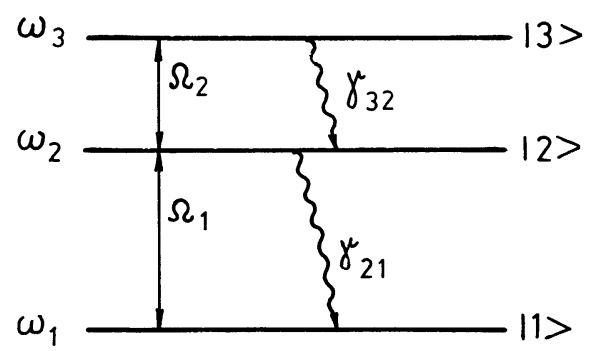

Fig. 1. - Three-level system of atoms interacting with two monochromatic applied fields and with emitted field.

(*) Permanent address : National Institute for Atomic Energy, 67 Nguyen Du, Hanoï, Viet-Nam. observed in the experimental works by Slusher et al. [20], Shelby et al. [21] and Kimble et al. [22].

In this paper we present the violation of the C-S inequality and the squeezing in the fluorescence from a system of three-level atoms (Fig. 1) interacting with two driving monochromatic resonant fields and with an emitted field in the context of double optical resonance [23-25].

\section{Master equation.}

The $N$ three-level atoms are assumed to be concentrated in a region small compared to the wavelength of all the relevant radiation modes. In treating the external fields as $C$-numbers, the master equation for the atomic system alone $\rho$ with the Markovian and rotating wave approximation is [27].

$$
\begin{aligned}
\frac{\partial \rho}{\partial t}= & -i G\left[\left(\cos \alpha J_{12}+\sin \alpha J_{23}+\text { H.C. }\right), \rho\right]- \\
& -\gamma_{21}\left(J_{21} J_{12} \rho-2 J_{12} \rho J_{21}+\rho J_{21} J_{12}\right) \\
& -\gamma_{32}\left(J_{32} J_{23} \rho-2 J_{23} \rho J_{32}+\rho J_{32} J_{23}\right) \equiv L \rho,
\end{aligned}
$$

where $2 \gamma_{21}$ and $2 \gamma_{32}$ are radiative spontaneous transition probabilities per unit time for a single atom to change from the level $|2\rangle$ to $|1\rangle$ and from $|3\rangle$ to $|2\rangle$, respectively ; $G=\left(G_{1}^{2}+G_{2}^{2}\right)^{1 / 2}$ and $\operatorname{tg} \alpha=G_{2} / G_{1}$ where $G_{1}$ and $G_{2}$ are the Rabi frequencies for the atomic transitions from level $|2\rangle$ to $|1\rangle$ and from $|3\rangle$ to $|2\rangle$ respectively; and

$$
J_{i j}=\sum_{K=1}^{N}|i\rangle_{K K}\langle j| \quad(i, j=1,2,3)
$$


are the collective angular momenta of the atoms. They satisfy the commutation relation

$$
\left[J_{i j}, J_{i^{\prime} j^{\prime}}\right]=J_{i j^{\prime}} \delta_{i^{\prime} j}-J_{i^{\prime} j} \delta_{i j^{\prime}} .
$$

As in references $[19,25,28]$, we introduce the Schwinger representation for angular momentum

$$
J_{i j}=C_{i}^{+} C_{j}, \quad(i, j=1,2,3),
$$

where the operators $C_{i}$ obey the boson commutation relation

$$
\left[C_{i}, C_{j}^{+}\right]=\delta_{i j}
$$

Furthermore, we investigate only the case of intense external fields so that

$$
G \gg N \gamma_{21}, N \gamma_{32}
$$

After performing the canonical transformation

$$
\begin{aligned}
C_{3}= & -\frac{1}{\sqrt{2}} \sin \alpha Q_{1}+\cos \alpha Q_{2}+ \\
& +\frac{1}{\sqrt{2}} \sin \alpha Q_{3}, \\
C_{2}= & \frac{1}{\sqrt{2}} Q_{1}+\frac{1}{\sqrt{2}} Q_{3}, \\
C_{1}= & -\frac{1}{\sqrt{2}} \cos \alpha Q_{1}-\sin \alpha Q_{2}+ \\
& +\frac{1}{\sqrt{2}} \cos \alpha Q_{3},
\end{aligned}
$$

one can find that the Liouville operator $L$ appearing in equation (1) splits into two components $L_{0}$ and $L_{1}$. The component $L_{0}$ is slowly varying in time whereas $L_{1}$ contains rapidly oscillating terms at frequencies $n G(n=1,2,3,4)$. For the case when relation (2) is fulfilled, we make the secular approximation, i.e., retain only a slowly varying part [25, 30]. Correction of the results obtained in this fashion will be on the order of $\left(\gamma_{21} N / G\right)^{2}$ or $\left(\gamma_{32} N / G\right)^{2}$.

Making the secular approximation, one can find the stationary solution of the master equation

$$
\tilde{\rho}=Z^{-1} \sum_{R=0}^{N} X^{R} \sum_{M=0}^{R}|M, R\rangle\langle R, M| .
$$

where $\tilde{\rho}=U \rho U^{+}$, here $U$ is the unitary operator representing the canonical transformation (3)

$$
\begin{gathered}
X=\frac{\gamma_{32} \cos ^{2} \alpha}{\gamma_{21} \sin ^{2} \alpha} ; \\
Z=\frac{(N+1) X^{N+2}-(N+2) X^{N+1}+1}{(X-1)^{2}} .
\end{gathered}
$$

The state $|M, R\rangle$ is an eigenstate of the operators $R_{11}, R=R_{11}+R_{33}$ and $\hat{N}=R_{11}+R_{22}+R_{33}$ where

$$
R_{i j}=Q_{i}^{+} Q_{j} \quad(i, j=1,2,3) .
$$

The operators $Q_{i}$ satisfy the boson commutation relation

$$
\left[Q_{i}, Q_{j}^{+}\right]=\delta_{i j}
$$

so that

$$
\left[R_{i j}, R_{i^{\prime} j^{\prime}}\right]=R_{i j^{\prime}} \delta_{i^{\prime} j}-R_{i^{\prime} j} \delta_{i j^{\prime}}
$$

By using solution (4) the characteristic function can be defined similarly to Louisell [29]

$$
\begin{aligned}
& \chi_{R}(\zeta)=\left\langle e^{i \zeta R}\right\rangle_{S}= \\
& =Z^{-1} \frac{(N+1) Y^{N+2}-(N+2) Y^{N+1}+1}{(Y-1)^{2}}
\end{aligned}
$$

where $Y=X \mathrm{e}^{i \zeta}$ and $\langle B\rangle_{S}$ indicates the expectation value of an operator $B$ in the steady state (4).

Once the characteristic function is known, it is easy to calculate the statistical moments

$$
\left\langle R^{n}\right\rangle_{S}=\left.\frac{\partial^{n}}{\partial(i \zeta)^{n}} \chi_{R}(\zeta)\right|_{i \zeta=0} .
$$

\section{Squeezing in the fluorescent light.}

In this section we discuss the squeezing in the fluorescence light in the collective double resonance process.

The variance of the fluctuations in the fluorescent field may be derived by using the following relations between the radiation field and the atomic operator in the far-field limit [16]

$$
\begin{aligned}
& E_{1}^{(+)}\left(\mathbf{X}, t^{\prime}\right)=E_{1, \text { free }}^{(+)}\left(\mathbf{X}, t^{\prime}\right)+\psi_{12}(\mathbf{X}) J_{12}(t) \mathrm{e}^{-i \Omega_{1} t} \\
& E_{1}^{(-)}\left(\mathbf{X}, t^{\prime}\right)=\left(E_{1}^{(+)}\left(\mathbf{X}, t^{\prime}\right)\right)^{+} \\
& E_{2}^{(+)}\left(\mathbf{X}, t^{\prime}\right)=E_{2, \text { free }}^{(+)}\left(\mathbf{X}, t^{\prime}\right)+\psi_{23}(\mathbf{X}) J_{23}(t) \mathrm{e}^{-i \Omega_{2} t} \\
& E_{2}^{(-)}\left(\mathbf{X}, t^{\prime}\right)=\left(E_{2}^{(+)}\left(\mathbf{X}, t^{\prime}\right)\right)^{+},
\end{aligned}
$$

where $t=t^{\prime}-r / c$,

$$
\begin{aligned}
& \psi_{12}(\mathbf{X})=\frac{\omega_{21}}{2 \pi \varepsilon_{0} c^{2}} \frac{\mathbf{X} \times\left(\mathbf{d}_{12} \times \mathbf{X}\right)}{r^{3}}, \\
& \psi_{23}(\mathbf{X})=\frac{\omega_{32}}{2 \pi \varepsilon_{0} c^{2}} \frac{\mathbf{X} \times\left(\mathbf{d}_{23} \times \mathbf{X}\right)}{r^{3}},
\end{aligned}
$$

the values of $\psi_{12}$ and $\psi_{23}$ are assumed to be real, d and $\mathbf{X}$ are the dipole vector of the atoms and the observation point vector, respectively ; $r=|\mathbf{X}|$; $E_{1}^{(+)}$and $E_{2}^{(+)}$are the positive-frequency parts of the fluorescent fields corresponding to the lower and upper atomic transitions $|2\rangle$ to $|1\rangle$ and $|3\rangle$ to $|2\rangle$, respectively.

From the canonical transformation (3), one can write the collective angular moments $J_{12}$ and $J_{23}$ in the form 


$$
\begin{aligned}
J_{12}=\frac{1}{2} \cos \alpha D_{3}+\frac{1}{2} \cos \alpha\left(R_{31}-R_{13}\right)- \\
-\frac{1}{\sqrt{2}} \sin \alpha\left(R_{23}+R_{21}\right),
\end{aligned}
$$

$$
\begin{aligned}
J_{23}=\frac{1}{2} \sin \alpha D_{3}+\frac{1}{2} & \sin \alpha\left(R_{31}-R_{13}\right)+ \\
& +\frac{1}{\sqrt{2}} \cos \alpha\left(R_{12}+R_{32}\right),
\end{aligned}
$$

where $D_{3}=R_{33}-R_{11}$.

Following the works $[29,16]$ we can consider the operators $\quad-\frac{1}{2} \cos \alpha R_{13}(t), \quad-\frac{1}{\sqrt{2}} \sin \alpha R_{23}(t)$, $\frac{1}{2} \cos \alpha D_{3}(t),-\frac{1}{\sqrt{2}} \sin \alpha R_{21}(t)$ and $\frac{1}{2} \cos \alpha R_{31}(t)$ as the amplitude-operators for the source of the spectrum components at the frequencies $\Omega_{1}-2 G$, $\Omega_{1}-G, \Omega_{1}, \Omega_{1}+G$ and $\Omega_{1}+2 G$; and for simplicity we denote these operators by $S_{-2}, S_{-1}$, $S_{0}, S_{1}$ and $S_{2}$, respectively.

Analogously, the operators $-\frac{1}{2} \sin \alpha R_{13}(t)$, $\frac{1}{\sqrt{2}} \cos \alpha R_{12}(t), \frac{1}{2} \sin \alpha D_{3}(t), \frac{1}{\sqrt{2}} \cos \alpha R_{32}(t)$ and $\frac{1}{2} \sin \alpha R_{31}(t)$ can be considered as the amplitude operators for the sources of the spectrum components at the frequencies $\Omega_{2}-2 G, \Omega_{2}-G, \Omega_{2}$, $\Omega_{2}+G$ and $\Omega_{2}+2 G$ and for simplicity we denote these operators by $T_{-2}, T_{-1}, T_{0}, T_{-1}$ and $T_{2}$, respectively.

Applying the relations (9)-(12) and steady-state solution (4), one can show that the squeezing is absent for the separate fields $E_{1}^{(+)}$and $E_{2}^{(+)}$. The squeezing is also absent for all separate spectral components $S_{i}$ and $T_{i}(i=0, \pm 1, \pm 2)$. The following calculations show that the squeezing exists only in the mixtures of two spectrum components $S_{1}$ and $T_{-1}$ or $S_{-1}$ and $T_{1}$; moreover, the degrees of squeezing in the mixture of $S_{1}$ and $T_{-1}$ and in the mixture of $S_{-1}$ and $T_{1}$ are equal. Further, we discuss only the squeezing in the mixture of two spectrum components $S_{-1}$ and $T_{+1}$. After substituting the operator $J_{12}$ in the relation (9) by the operator $S_{-1}=-\frac{1}{\sqrt{2}} \sin \alpha R_{23}$ and the operator $J_{23}$ in relation (10) by the operator $T_{1}=\frac{1}{\sqrt{2}} \cos \alpha R_{32}$ and using the steady-state solution (4) one finds the normally ordered variance of fluctuations for the Hermitian amplitude operators of the mixture of two spectrum components $S_{-1}$ and $T_{1}$ in the form

$$
\begin{aligned}
\left\langle:\left(\Delta a_{1,2}\right)^{2}:\right\rangle & =\frac{1}{4}\left\{\psi_{12}^{2} \sin ^{2} \alpha\left\langle R_{32} R_{23}\right\rangle_{s}+\right. \\
+\psi_{23}^{2} \cos ^{2} \alpha & \left\langle R_{23} R_{32}\right\rangle_{s} \mp \psi_{12} \psi_{23} \cos \alpha \cdot \sin \alpha \times \\
& \times\left(\left\langle R_{32} R_{23}\right\rangle_{s}+\left\langle R_{23} R_{32}\right\rangle\right.
\end{aligned}
$$

where

$$
\begin{array}{ll}
a_{1}=\frac{1}{2}\left(a^{+}+a\right), & a_{2}=\frac{-i}{2}\left(a-a^{+}\right) \\
a=\tilde{E}_{1}^{(+)}+\tilde{E}_{2}^{(+)}, & a^{+}=\tilde{E}_{1}^{(-)}+\tilde{E}_{2}^{(-)}
\end{array}
$$

the operators $\tilde{E}_{1}^{( \pm)}$and $\tilde{E}_{2}^{( \pm)}$are derived from $E_{1}^{( \pm)}$and $E_{2}^{( \pm)}$by the replacement in relations (9) and (10) of the operators $J_{12}$ and $J_{23}$ by the operators $S_{-1}$ and $T_{+1}$, respectively.

The statistical moments $\left\langle R_{32} R_{23}\right\rangle_{s}$ and $\left\langle R_{23} R_{32}\right\rangle_{s}$ can be written in the form

$$
\begin{aligned}
& \left\langle R_{23} R_{32}\right\rangle_{s}=\frac{1}{2}(N-2)\langle R\rangle_{s}-\frac{\left\langle R^{2}\right\rangle_{s}}{2}+N, \\
& \left\langle R_{32} R_{23}\right\rangle_{S}=\frac{1}{2}(N+1)\langle R\rangle_{s}-\frac{1}{2}\left\langle R^{2}\right\rangle_{S},
\end{aligned}
$$

here $\langle R\rangle_{S}$ and $\left\langle R^{2}\right\rangle_{S}$ can be found in relation (8). We speak of squeezing in the mixture of two spectrum components $S_{-1}$ and $T_{1}$ if the normally ordered variance of the operators $a_{1}$ or $a_{2}$ is less than zero [16-18]

$$
\left\langle:\left(\Delta a_{i}\right)^{2}:\right\rangle<0 \quad(i=1 \text { or } 2) .
$$

It is easy to see that in the case of $X=1$, we have $\left\langle R_{23} R_{32}\right\rangle_{S}=\left\langle R_{32} R_{23}\right\rangle_{S}$ and the relation (13) reduces to

$$
\begin{aligned}
\left\langle:\left(\Delta a_{1,2}\right)^{2}:\right\rangle & =\left\langle R_{23} R_{32}\right\rangle_{s} \times \\
& \times\left(\psi_{12} \sin \alpha \mp \psi_{23} \cos \alpha\right)^{2} \geqslant 0
\end{aligned}
$$

thus, the squeezing is absent in this case. It is also easy to see from the relation (13) that the squeezing is absent in the case $\operatorname{ctg} \alpha \rightarrow 0$ or $\operatorname{ctg} \alpha \rightarrow \infty$.

The detailed behaviour of $\left\langle:\left(\Delta a_{1}\right)^{2}:\right\rangle$ (in the relative unity $\left.\frac{1}{4} \psi_{12}^{2}\right)$ as a function of the parameter $\operatorname{ctg}^{2} \alpha$ in the case $\gamma_{32}=\gamma_{21}$ and $\psi_{12}=\psi_{23}>0$ is plotted in figure 2 for various numbers of atoms. As shown in figure 2, a substantial squeezing occurs for the mixture of two spectrum components $S_{-1}$ and $T_{1}$ and the optimum of squeezing appears in the region of the parameter $\operatorname{ctg}^{2} \alpha<1$. As it has been already mentioned above the squeezing is absent for all separate spectral components $S_{i}$ and $T_{i}(i=0$, $\pm 1, \pm 2)$ and for the fields $E_{1}^{(+)}$and $E_{2}^{(+)}$taken separately.

Analogously to the work [17], we can define the factor of squeezing for the atomic operators

$$
A_{1}=\frac{1}{2}\left(A+A^{+}\right), \quad A_{2}=-\frac{i}{2}\left(A-A^{+}\right)
$$

where

$$
A=\dot{\psi}_{12} S_{-1}+\psi_{23} T_{1}
$$



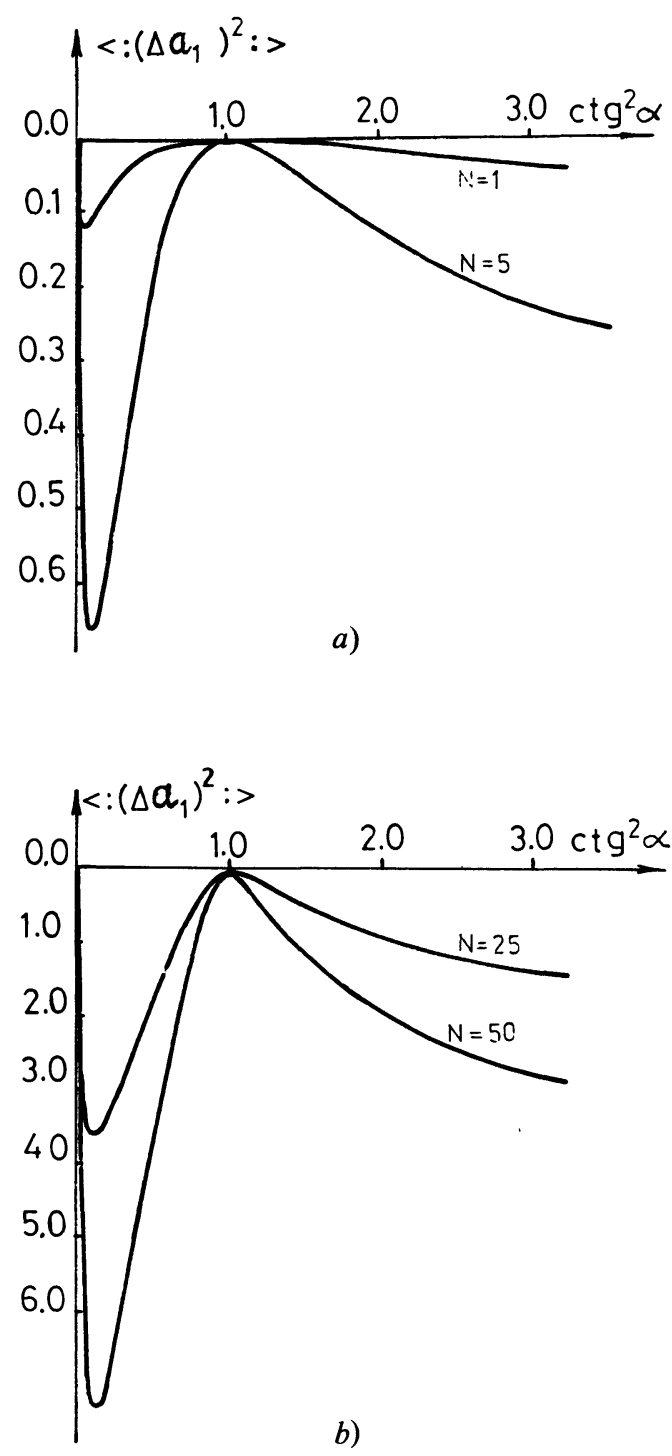

Fig. 2. - (a-b) Normally ordered variance $\left\langle:\left(\Delta a_{1}\right)^{2}:\right\rangle$ (in the relative unity $\frac{1}{4} \psi_{12}^{2}$ ) as a function of $\operatorname{ctg}^{2} \alpha$ for the case of $\gamma_{32}=\gamma_{21}$ and $\psi_{12}=\psi_{23}>0$.

in the form

$F_{1,2}=\frac{\left\langle:\left(\Delta A_{1,2}\right)^{2}:\right\rangle_{s}}{\frac{1}{2}\left|\left\langle\left[A_{1}, A_{2}\right]\right\rangle_{s}\right|}=\frac{\left\langle\left(\Delta A_{1,2}\right)^{2}\right\rangle_{s}}{\frac{1}{2}\left|\left\langle\left[A_{1}, A_{2}\right]\right\rangle_{s}\right|}-1$.

The behaviour of the factor of squeezing $F_{1}$ as a function of $\operatorname{ctg}^{2} \alpha$ in the case $\gamma_{32}=\gamma_{21}$, $\psi_{12}=\psi_{23}>0$ is plotted in figure 3. As is shown in figure 3 the factors of squeezing for atomic operator $A_{1}$ are independent of the number of atoms and can tend to the value $F_{1}=-0.5$ (i.e. the $50 \%$ of squeezing) in a region around the point $\operatorname{ctg}^{2} \alpha=1$. One can show that $\left[A_{1}, A_{2}\right]=0$ when $\operatorname{ctg}^{2} \alpha=1$. Thus in this case, even if $F_{1}=-0.5$, the concept of

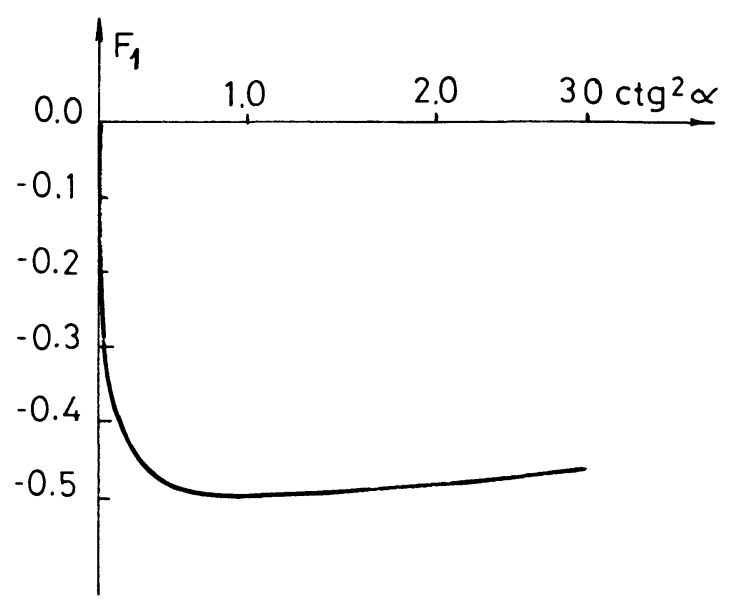

Fig. 3. - Factor of squeezing $F_{1}$ as a function of parameter $\operatorname{ctg}^{2} \alpha$ for the case of $\gamma_{32}=\gamma_{21}$ and $\psi_{12}=\psi_{23}>0$.

squeezing for the commutating operators $A_{1}$ and $A_{2}$ loses sense.

We note that though

$$
\left\langle:\left(\Delta A_{i}\right)^{2}:\right\rangle_{s}=\left\langle:\left(\Delta a_{i}\right)^{2}:\right\rangle, \quad(i=1,2)
$$

as a result of the influence of the free parts of the fluorescent fields $E_{1, \text { free }}^{( \pm)}$and $E_{2, \text { free }}^{( \pm)}$(see relations (9)(10)) the factors of squeezing $F_{1}, F_{2}$ for the atomic operators $A_{1}, A_{2}$ are not coincident with the factors of squeezing for the field operators $a_{1}$ and $a_{2}$.

\section{Violation of the Cauchy-Schwarz (C-S) inequality.}

Analogously to [7], we define a degree of secondorder coherence between the spectrum components $S_{i}$ and $S_{j}$ in the form

$G_{i, j}^{(2)}=\frac{\left\langle S_{i}^{+} S_{j}^{+} S_{j} S_{i}\right\rangle_{s}}{\left\langle S_{i}^{+} S_{i}\right\rangle_{S}\left\langle S_{j}^{+} S_{j}\right\rangle_{S}} \quad(i, j=0, \pm 1, \pm 2)$.

In a general case the operators $S_{i}$ and $S_{j \neq i}^{+}$do not commute and we have $G_{i, j}^{(2)} \neq G_{j, i}^{(2)}$.

The photon antibunching is exhibited for the spectrum component $S_{i}(i=0, \pm 1, \pm 2)$ which satisfy the inequality

$$
G_{i, i}^{(2)}<1,
$$

i.e. the degree of second-order coherence is less than unity. Such properties occurring for the four sidebands $S_{i}(i= \pm 1, \pm 2)$ in the case of one or several atoms are investigated in the work [19].

Further, we shall discuss another non-classical effect : the violation of the C-S inequality in the stationary fluorescent field.

We speak about the violation of the C-S inequality for the correlation between two spectrum compo- 
nents $S_{i}$ and $S_{j}(i, j=0, \pm 1, \pm 2)$ if the following condition is satisfied [33] :

$$
K_{i, j}=\frac{G_{i, j}^{(2)} \cdot G_{j, i}^{(2)}}{\left(G_{i, j}^{(2)}\right)^{2}}<1
$$

The factor $K_{i, j}$ describes the degree of violation of the C-S inequality for the correlation between two spectrum components $S_{i}$ and $S_{j}$ by using the commutation relations (5)-(6) and the stationary solution (4), one can find:

$$
\begin{gathered}
K_{2,-2}=K_{-2,2}=\frac{\left(\left\langle R_{31} R_{31} R_{13} R_{13}\right\rangle_{s}\right)^{2}}{\left(\left\langle R_{31} R_{13} R_{31} R_{13}\right\rangle_{s}\right)^{2}}, \\
K_{0,2}=K_{2,0}=K_{0,-2}=K_{-2,0}=\frac{\left\langle R_{31} R_{31} R_{13} R_{13}\right\rangle_{s} \cdot\left\langle D_{3}^{4}\right\rangle}{\left(\left\langle D_{3} R_{31} R_{13} D_{3}\right\rangle_{s}\right)^{2}}, \\
K_{0,1}=K_{0,-1}=\frac{\left\langle R_{32} R_{32} R_{23} R_{23}\right\rangle_{s} \cdot\left\langle D_{3}^{4}\right\rangle_{s}}{\left(\left\langle D_{3} R_{32} R_{23} D_{3}\right\rangle_{s}\right)^{2}}, \\
K_{2,-1}=K_{-2,1}=\frac{\left\langle R_{31} R_{31} R_{13} R_{13}\right\rangle_{s} \cdot\left\langle R_{12} R_{12} R_{21} R_{21}\right\rangle_{s}}{\left(\left\langle R_{31} R_{12} R_{21} R_{13}\right\rangle_{s}\right)^{2}}, \\
K_{-1,2}=K_{1,-2}=\frac{\left\langle R_{31} R_{31} R_{13} R_{13}\right\rangle_{s} \cdot\left\langle R_{12} R_{12} R_{21} R_{21}\right\rangle_{s}}{\left(\left\langle R_{12} R_{31} R_{13} R_{21}\right\rangle_{s}\right)^{2}}
\end{gathered}
$$

where

$$
\left\langle D_{3}^{4}\right\rangle_{S}=\frac{1}{15}\left(3\left\langle R^{4}\right\rangle_{S}+12\left\langle R^{3}\right\rangle_{S}+8\left\langle R^{2}\right\rangle_{S}-8\langle R\rangle_{S}\right),
$$

$$
\begin{aligned}
& \left\langle R_{12} R_{12} R_{21} R_{21}\right\rangle_{S}=\frac{1}{3}\left[\left\langle R^{4}\right\rangle_{S}-2(N+2)\left\langle R^{3}\right\rangle_{S}+\left(N^{2}+5 N+5\right)\left\langle R^{2}\right\rangle_{S}-(N+1)(N+2)\langle R\rangle_{S}\right], \\
& \left\langle R_{31} R_{31} R_{13} R_{13}\right\rangle_{S}=\frac{1}{30}\left(\left\langle R^{4}\right\rangle_{S}+4\left\langle R^{3}\right\rangle_{S}+\left\langle R^{2}\right\rangle_{S}-6\langle R\rangle_{S}\right) \\
& \left\langle R_{31} R_{13} R_{31} R_{13}\right\rangle_{s}=\frac{1}{12}\left(-\left\langle R^{4}\right\rangle_{s}+N\left\langle R^{3}\right\rangle_{s}+(N+3)\left\langle R^{2}\right\rangle_{s}-2(N+1)\langle R\rangle_{s}\right), \\
& \left\langle D_{3} R_{31} R_{13} D_{3}\right\rangle_{s}=\frac{1}{30}\left(\left\langle R^{4}\right\rangle_{s}+4\left\langle R^{3}\right\rangle_{S}+6\left\langle R^{2}\right\rangle_{S}+4\langle R\rangle_{S}\right), \\
& \left\langle D_{3} R_{32} R_{23} D_{3}\right\rangle_{s}=\frac{1}{6}\left(-\left\langle R^{4}\right\rangle_{s}+(N-1)\left\langle R^{3}\right\rangle_{s}+2(N+1)\left\langle R^{2}\right\rangle_{s}\right), \\
& \left\langle R_{31} R_{12} R_{21} R_{13}\right\rangle_{s}=\frac{1}{12}\left(-\left\langle R^{4}\right\rangle_{s}+(N+2)\left\langle R^{3}\right\rangle_{s}+(3 N+1)\left\langle R^{2}\right\rangle_{s}+2(N+1)\langle R\rangle_{s}\right), \\
& \left\langle R_{12} R_{31} R_{13} R_{21}\right\rangle_{S}=\frac{1}{12}\left(-\left\langle R^{4}\right\rangle_{S}+N\left\langle R^{3}\right\rangle_{S}+(N+3)\left\langle R^{2}\right\rangle_{S}\right) .
\end{aligned}
$$

Further calculations show that the other factors are more than unity for any number of atoms :

$$
\begin{aligned}
K_{1,-1} & =K_{-1,1}>1, \\
K_{2,1} & =K_{1,2}=K_{-1,-2}=K_{-2,-1}>1, \\
K_{1,0} & =K_{-1,0}>1 .
\end{aligned}
$$

It means that the classical C-S inequality occurs in these cases.

The behaviour of the functions $K_{2,-2}, K_{0,2}$, $K_{0,1}, K_{2,-1}$ and $K_{-1,2}$ against the parameter $X$ is shown in figure 4 (a-e) for : various numbers of atoms. It is easy to see from figure 4 (a-e) that the strong violation of the C-S inequality exists for a large number of atoms. It means that in contrast with the effect of photon antibunching, the violation of the C-S inequality is a macroscopic quantum effect.

To conclude, we note that the investigation of the violation of the C-S inequality for the correlations between the spectra components corresponding to the upper atomic transition $|3\rangle \rightarrow|2\rangle$ and between spectra of the upper and lower atomic transitions can be carried out by using an analogous approach. 


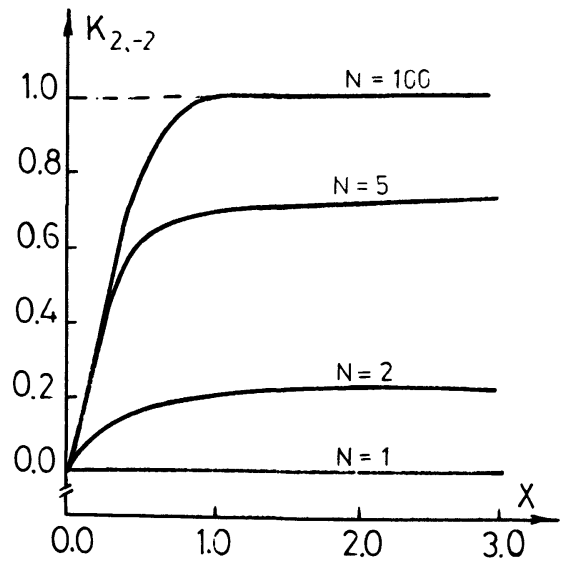

a)

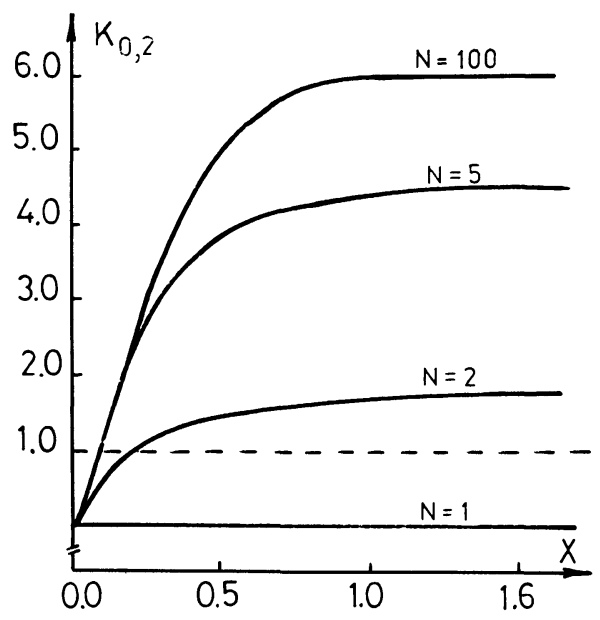

b)

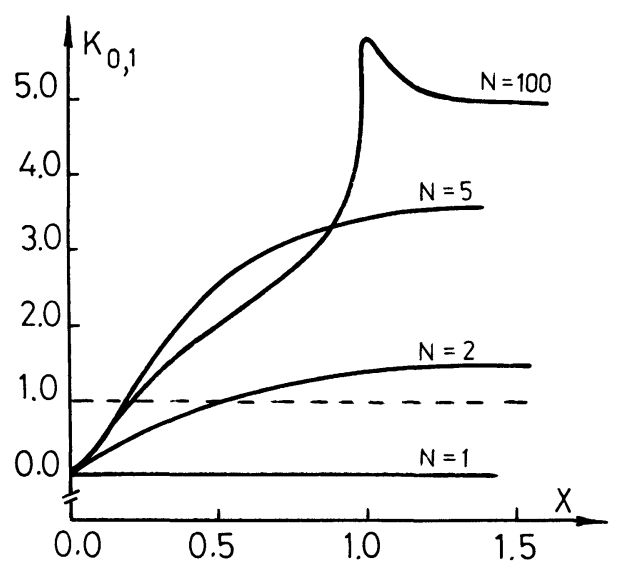

c)

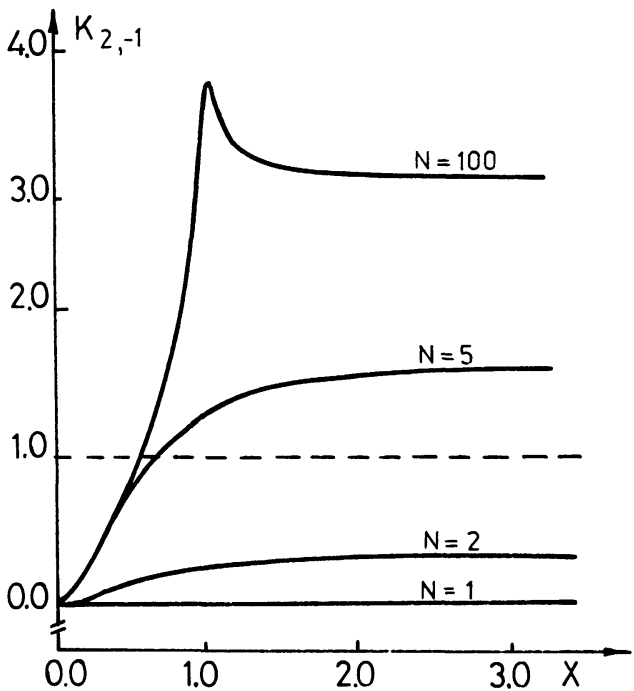

d)

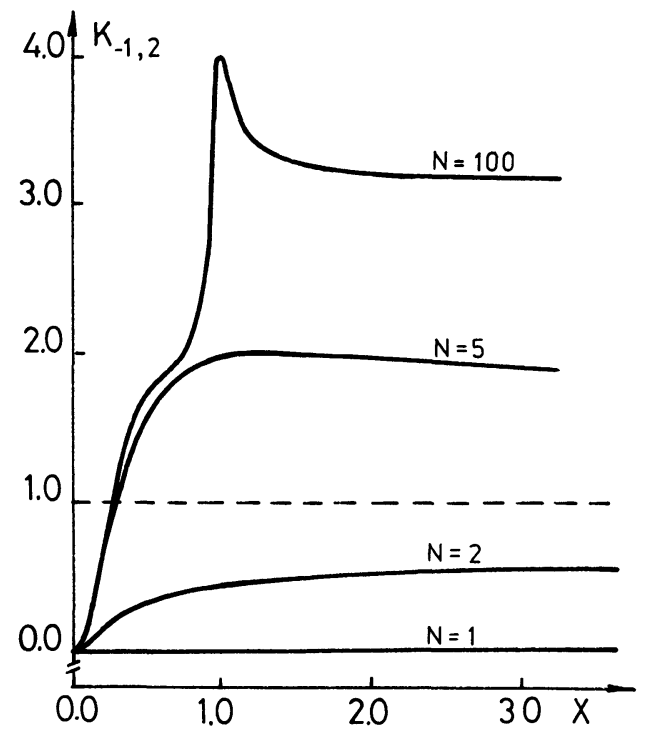

e)

Fig. 4. - (a-e) Factors $K_{i, j}$ as functions of parameter $X$. 


\section{References}

[1] Loudon, R., Rep. Prog. Phys. 43 (1980) 38.

[2] Paul, H., Rev. Mod. Phys. 54 (1982) 1061.

[3] Walls, D. F., Nature 306 (1983) 141.

[4] Kimble, H. J., Dagenais, M. and Mandel, L., Phys. Rev. Lett. 39 (1977) 691.

[5] Kimble, H. J., Dagenais, M. and Mandel, L., Phys. Rev. A 18 (1978) 201.

[6] Clauser, F., Phys. Rev. D 9 (1974) 853.

[7] Zubairy, M. S., Phys. Lett. A 87 (1982) 162.

[8] McNeil, K. J. and.Gardiner, C. W., Phys. Rev. A 28 (1983) 1560.

[9] Yuen, H. P., Phys. Rev. A 13 (1976) 2226.

[10] Lugiato, L. A. and Strini, G., Opt. Commun. 41 (1982) 67.

[11] Yurke, B., Phys. Rev. A 29 (1984) 408.

[12] Collet, M. J., Gardiner, C. W., Phys. Rev. A 30 (1984) 1386.

[13] Bondurant, R. S., Kumar, P., Shapiro, S. H. and Maeda, M., Phys. Rev. A 30 (1984) 343.

[14] ReId, M. D. and Walls, P. F., Phys. Rev. A 31 (1985) 1622.

[15] Mandel, L., Phys. Rev. Lett. 49 (1982) 136.

[16] Walls, D. F. and Zoller, P., Phys. Rev. Lett. 47 (1981) 709.

[17] Lakshmi, P. A. and Agarwal, G. S., Opt. Commun. 51 (1984) 425.

[18] Lakshmi, P. A. and Agarwal, G. S., Phys. Rev. A 29 (1984) 2260.

[19] Bogolubov (Jr.), N. N., Shumovsky, A. S. and Tran Quang, Phys. Lett. A 122 (1987) 25.

[20] Slusher, R. E., Hollberg, L. W., Yurke, B., Mertz, J. C. and Valley, J. F., Phys. Rev. Lett. 55 (1985) 2409.
[21] Shelby, R. M., Levenson, M. D., Perlmutter, S. H., Devoe, R. G. and Walls, D. F., Phys. Rev. Lett. 57 (1986) 691.

[22] Wu, Ling-An, Kimble, H. J., Hall, J. L. and Wu, Huifa, Phys. Rev. Lett. 57 (1986) 2520.

[23] Cohen-Tannoudj, C. and Reynaud, S., J. Phys. B 10 (1977) 2311.

[24] Whitley, R. M. and Stroud (Jr.), C. R., Phys. Rev. A 14 (1976) 1498.

[25] Bogolubov (Jr.), N. N., Shumovsky, A. S. and Tran Quang, Phys. Lett. A 112 (1985) 323.

[26] Apanasevich, P. A. and Killin, S. J., J. Phys. B 23 (1979) L83.

[27] Agarwal, G. S., Springer Tracts in modern Physics, 70 (Berlin-New York) 1974.

[28] SCHWInger, J., Quantum Theory of Angular Momentum, Biederharn L. C. and Van Dam H., Eds., New York, 1965.

[29] Louisell, W. H., Radiation and Noise in quantum electronies (Mc Graw-Hill book company, New York) 1964.

[30] Agarwal, G. S., Narducci, L. M., Da Hsuan Feng and Gilmore, R., Phys. Rev. Lett. 42 (1978) 1260.

[31] Kozierowski, M., Phys. Rev. A 34 (1986) 3474.

[32] Ficek, Z., TANas, R. and Kielich, S., Phys. Rev. A 29 (1984) 2004.

[33] Reid, M. D. and Walls, D. F., Phys. Rev. A 34 (1986) 1260. 